\title{
Thermal decomposition of RDX from reactive molecular dynamics
}

\author{
Alejandro Strachan a) and Edward M. Kober \\ Theoretical Division, Los Alamos National Laboratory, Los Alamos, New Mexico 87545 \\ Adri C. T. van Duin, Jonas Oxgaard, and William A. Goddard III \\ Materials and Process Simulation Center, Beckman Institute (139-74), California Institute of Technology, \\ Pasadena, California 91125
}

(Received 3 August 2004; accepted 21 October 2004; published online 13 January 2005)

\begin{abstract}
We use the recently developed reactive force field ReaxFF with molecular dynamics to study thermal induced chemistry in RDX [cyclic- $\left.\left[\mathrm{CH}_{2} \mathrm{~N}\left(\mathrm{NO}_{2}\right)\right]_{3}\right]$ at various temperatures and densities. We find that the time evolution of the potential energy can be described reasonably well with a single exponential function from which we obtain an overall characteristic time of decomposition that increases with decreasing density and shows an Arrhenius temperature dependence. These characteristic timescales are in reasonable quantitative agreement with experimental measurements in a similar energetic material, $\mathrm{HMX}$ [cyclic- $\left.\left[\mathrm{CH}_{2} \mathrm{~N}\left(\mathrm{NO}_{2}\right)\right]_{4}\right]$. Our simulations show that the equilibrium population of $\mathrm{CO}$ and $\mathrm{CO}_{2}$ (as well as their time evolution) depend strongly of density: at low density almost all carbon atoms form $\mathrm{CO}$ molecules; as the density increases larger aggregates of carbon appear leading to a $\mathrm{C}$ deficient gas phase and the appearance of $\mathrm{CO}_{2}$ molecules. The equilibrium populations of $\mathrm{N}_{2}$ and $\mathrm{H}_{2} \mathrm{O}$ are more insensitive with respect to density and form in the early stages of the decomposition process with similar timescales. (C) 2005 American Institute of Physics. [DOI: 10.1063/1.1831277]
\end{abstract}

\section{INTRODUCTION}

A molecular level understanding of condensed-matter chemistry is a central problem in many areas of chemistry and materials science. Despite the enormous experimental and theoretical progress in recent years a wide variety of phenomena remains uncharacterized. Particularly challenging is understanding the decomposition and subsequent reactions of high energy (HE) materials due to the extreme conditions of temperature and pressure involved as well as their complex chemistry. Recent advances in experimental techniques such as ultra-fast spectroscopy ${ }^{1}$ and interferometry ${ }^{2}$ are beginning to provide molecular-level information about the decomposition of HE materials. Nevertheless, molecular dynamics (MD) remains the only technique capable of providing the spatial and temporal resolution necessary to characterize a variety of fundamental issues where a detailed understanding is still lacking, such as the initial chemical steps of decomposition, detailed chemical reaction pathways (uniand multi-molecular), and the molecular structure of products. Such fundamental information is necessary for the development of physics-based, predictive materials models that can be used in large-scale macroscopic simulations of HE materials.

The fundamental input in MD simulations are the atomic interactions, which are described from first principles by quantum mechanics (QM). Unfortunately QM methods are computationally too intensive to describe most processes of interest under realistic conditions (especially when chemical reactions need to be described). This limitation has been overcome, to a large extent, in recent years with the devel-

${ }^{a)}$ Electronic mail: strachan@lanl.gov opment of reactive force fields (FFs) that can describe chemical reactions in a computationally efficient way. ${ }^{3-5}$ ReaxFF $^{4}$ is a new-generation bond order-dependent reactive FF based on extensive $a b$ initio QM calculations that enables for the first time the accurate simulation of HE materials under realistic conditions in a computationally efficient way.

Typical HE materials, such as TNT, RDX, HMX, TATB, and PETN, are complex organic molecular solids; their detonation involves the interplay between the mechanical shock loading, the induced thermo-mechanical response (for instance, the coupling between lattice modes and internal molecular modes, large internal deformations of the molecules, and plastic deformation) and the induced chemistry. The understanding of detonation was advanced significantly with the development of the ZND model by Zeldovich, von Neumann, and Doring during World War II. ${ }^{6}$ The leading shock front compresses and heats up the unreactive material. For strong enough shock waves the high temperatures and pressures trigger the chemical decomposition of the HE material and is followed by a decrease in density and pressure as the chemical reactions progress and the material expands in the reaction zone. ${ }^{6}$ For steady detonations the reaction zone moves with the leading shock at the detonation velocity and ends in the so-called Chapman-Jouget (CJ) point; a Taylor release wave follows, where the products expand and the pressure decreases to a value that depends on the rear boundary condition (equal to zero for unsupported detonations). A necessary condition for the steady propagation of a detonation is the existence of a "sonic point" that isolates the reaction zone from the release wave. The local sound speed decreases from right behind the shock front into the reaction zone and Taylor wave due to expansion; at the sonic point the local sound speed is equal to the detonation velocity such 
that no perturbation in the Taylor release wave can affect the reaction zone. ${ }^{6}$ Real HE formulations are defective (plastic bonded, porous, etc.) and these heterogeneities lead to hotspots and a complex multi-dimensional flow even in macroscopically unidirectional detonation.

MD with reactive potentials that exhibit simple, fast, and exothermic chemistry has been extremely useful to characterize detonation in model systems. Nonequilibrium MD shock simulations with these potentials enabled the atomistic description of steady detonation waves, ${ }^{7,8}$ and have been used to study the role of defects on initiation, ${ }^{9,10}$ the existence of a failure diameter, ${ }^{11}$ desensitization, ${ }^{12}$ etc. The direct observation of steady detonations is possible because these model potentials are tuned to have a very short reaction zone (around $10 \mathrm{~nm}$ ). In most high energy materials (including RDX, HMX, and TATB) the reaction zones are much wider (up to millimeters) and reaction times much longer (a fraction of a microsecond), making direct, nonequilibrium atomistic simulations of steady detonations impossible with current or near-future computational capabilities. Nevertheless reactive MD simulations using ReaxFF provided important information regarding the initial chemical events in RDX under shock loading. ${ }^{5} \mathrm{NO}_{2}$ was found to be the only product in weak shocks in agreement with experiments and, despite the fact that the barriers for pathways leading to $\mathrm{NO}_{2}$ and HONO are essentially the same, we also found that for strong shocks the primary reactions leading to $\mathrm{NO}_{2}, \mathrm{OH}$, $\mathrm{NO}$, and $\mathrm{N}_{2}$ occur at very early stages with time scales of a few ps.

Equilibrium MD simulations can provide valuable information regarding the thermal decomposition of HE materials enabling the characterization of processes with time scales much longer than those attainable in nonequilibrium shockwaves. For example, Manaa and collaborators ${ }^{13}$ used QMbased MD to study HMX decomposition at a temperature and density $\left(T=3500 \mathrm{~K}\right.$ and $\left.\rho=1.9 \mathrm{~g} / \mathrm{cm}^{3}\right)$ close to CJ conditions. Their simulations shed light into the initial chemical reactions and time scales for the production of several products. Unfortunately, the computational cost of such QM methods hinders their applicability to study decomposition at lower temperatures that require much longer simulation times (several nanoseconds). Another promising avenue to explore long time-scale phenomena with MD is the use of "hugoniostat" techniques, where the equations of motion of the atoms are modified to reproduce the state of the material caused by the passage of a shockwave. ${ }^{14-17}$

In this paper we use ReaxFF with MD to study thermalinduced decomposition of RDX at various densities (from $\rho=2.11 \mathrm{~g} / \mathrm{cm}^{3}$ in compression to $0.21 \mathrm{~g} / \mathrm{cm}^{3}$ in expansion) and over a wide temperature range (from $T=1200$ to 3000 $\mathrm{K})$. Our most extreme simulations $\left(\rho=2.11 \mathrm{~g} / \mathrm{cm}^{3}\right.$ and $T$ $=3000 \mathrm{~K})$ correspond to conditions close to those of the CJ point (final state of detonation) of RDX. In Sec. II we briefly describe ReaxFF and its parametrization. Section III describes our MD experiments on thermal decomposition and the molecule recognition method we used to analyze the simulations. In Secs. IV-VI, we present the results and their implications. Finally, in Sec. VII conclusions are drawn.

\section{ReaxFF FORCE FIELD}

In this paper we used the ReaxFF reactive force field for nitramines $\left(\operatorname{ReaxFF}_{\text {nitro }}\right)$ as previously used to study the initial chemical events in RDX during shock simulations. ${ }^{5}$ The potential functions in $\mathrm{ReaxFF}_{\text {nitro }}$ are very similar to those earlier reported for the hydrocarbon reactive force field. ${ }^{4}$ Here we will only describe the general concepts of ReaxFF, followed by a more detailed discussion of the differences between ReaxFF ${ }_{\text {nitro }}$ and $\operatorname{ReaxFF}_{C H}$.

$\mathrm{ReaxFF}_{\text {nitro }}$ partitions the system energy into the contributions described in Eq. (1):

$$
\begin{aligned}
E_{\text {total }}= & E_{\text {bond }}+E_{\text {over }}+E_{\text {under }}+E_{\text {lp }}+E_{\text {triple }}+E_{\text {val }}+E_{\text {pen }} \\
& +E_{\text {tors }}+E_{\text {coa }}+E_{\text {conj }}+E_{v d W}+E_{\text {Cou }} .
\end{aligned}
$$

A fundamental difference between the ReaxFF and unreactive force fields is that ReaxFF does not use fixed connectivity assignments for the chemical bonds. Instead the bond order, $\mathrm{BO}^{\prime}$, is calculated directly from the instantaneous interatomic distances $r_{i j}$ [see Eq. (2)], which are updated continuously during the dynamics. This allows for the creation and dissociation of bonds during a simulation. These instantaneous bond orders $\mathrm{BO}^{\prime}$ are corrected for overcoordination of the atoms sharing the bond, yielding corrected bond orders $\mathrm{BO}$ that are used subsequently on all covalent interactions [e.g., the functions describing bonds $\mathrm{E}_{\text {bond }}$, bond angles $\left(\mathrm{E}_{\text {angle }}\right)$ and torsions $\left.\left(\mathrm{E}_{\text {tors }}\right)\right]$ :

$$
\begin{aligned}
\mathrm{BO}_{i j}^{\prime}= & \mathrm{BO}_{i j}^{\prime \sigma}+\mathrm{BO}_{i j}^{\prime \pi}+\mathrm{BO}_{i j}^{\prime \pi \pi} \\
= & \exp \left[p_{\sigma}\left(\frac{r_{i j}}{r_{0}^{\sigma}}\right)_{\sigma}^{q}\right]+\exp \left[p_{\pi}\left(\frac{r_{i j}}{r_{0}^{\pi}}\right)_{\pi}^{q}\right] \\
& +\exp \left[p_{\pi \pi}\left(\frac{r_{i j}}{r_{0}^{\pi \pi}}\right)_{\pi \pi}^{q}\right] .
\end{aligned}
$$

All covalent interactions are expressed in terms of these bond orders so that these terms dissociate properly as any bond is broken. Since bonds can break and form during dynamics, we cannot exclude Coulomb and van der Waals interactions for bonded atoms as commonly done in many FF. Instead we must include these interactions between every atom pair, independent of the instantaneous connectivity. This is very natural for the Coulomb interactions since ReaxFF treats the atomic charges as having the finite size of the atom, leading to shielding of the Coulomb interaction for shorter distances. We also include a similar shielding for van der Waals interactions, reducing repulsion at short interatomic distances (see Eq. 12 in Ref. 4).

\section{A. New terms and modifications in ReaxFF}

To enable the description of nitramine chemistry the following additions were made to $\operatorname{ReaxFF}_{C H}$ :

Bond energy function. Both $\operatorname{ReaxFF}_{C H}$ and ReaxFF ${ }_{n i t r o}$ allow separate functional forms $\left(\mathrm{BO}_{\sigma}, \mathrm{BO}_{\pi}\right.$, and $\mathrm{BO}_{\pi \pi}$ ) to describe single, double, and triple bonds. Each of these bond orders has a different dependence on bond distance with the parameters determined from calculations on molecules such as $\mathrm{H} 3 \mathrm{C}-\mathrm{NH} 3, \mathrm{H} 2 \mathrm{C}=\mathrm{NH}$ and $\mathrm{HC} \equiv \mathrm{N}$. 
However, in ReaxFF $\mathrm{CH}_{\mathrm{H}}$ the total bond energy is described as a function only of the total bond order $\left(\mathrm{BO}_{\sigma}+\mathrm{BO}_{\pi}\right.$ $\left.+\mathrm{BO}_{\pi \pi}\right)$. In contrast ReaxFF $\mathrm{Fitro}_{\text {no }}$ uses separate energies for single, double, and triple bonds, as shown in Eq. (3). The reason for this change is that while carbon-carbon systems have bond energies that increase systematically for single to double to triple bonds (dissociation energies of 98, 178, 235 $\mathrm{kcal} / \mathrm{mol}$ ), multiple bonds in systems containing $\mathrm{N}$ and $\mathrm{O}$ can have dramatically different behaviors as bond orders increase. This arises because of the role in the bonding of lone pairs on $\mathrm{N}$ and $\mathrm{O}$, which can participate in bonding. Thus ReaxFF has been extended to partition the total bond energy into individual contributions from sigma-bonds, pi-bonds, and double pi-bonds (triple bonds), as indicated in Eq. (3). As in $\operatorname{ReaxFF}_{C H}$, the bond orders $\mathrm{BO}^{\prime}$ as obtained from Eq. (2) are corrected for overcoordination. Using these corrected contributions (BO), Eq. (3) is used to calculate bond energies in $\mathrm{ReaxFF}_{\text {nitro }}$ :

$E_{\text {bond }}=\sum_{i j}-D^{\sigma} B O_{i j}^{\sigma}+-D^{\pi} B O_{i j}^{\pi}+-D^{\pi \pi} B O_{i j}^{\pi \pi}$.

Lone pair electrons. The second new potential function used in ReaxFF nitro $_{\text {is }}$ an energy term associated with lone electron pairs $\left[E_{l p}\right.$ in Eq. (1)]. Lone pairs of electrons generally play little role in hydrocarbon chemistry, but lone pairs on such heteroatoms as oxygen and nitrogen are important in the response of these atoms to over- and undercoordination. Furthermore, the presence of these lone electron pairs influences the valence angles around atoms (this aspect was already incorporated in $\mathrm{ReaxFF}_{C H}$ ). In addition, lone pairs contribute to the stability of conjugated systems by delocalizing over adjacent pi bonds. Thus Eq. (4) is used to determine the number of lone pairs around an atom. $\Delta_{i}^{e}$ in Eq. (4) is the difference between the total number of outer shell electrons ( 6 for oxygen, 4 for silicon, 1 for hydrogen) and the sum of bond orders around an atomic center.

$$
n_{l p, i}=\operatorname{int}\left(\frac{\Delta_{i}^{e}}{2}\right)+\exp \left[-\lambda_{16}\left[2+\Delta_{i}^{e}-2 \operatorname{int}\left(\frac{\Delta_{i}^{e}}{2}\right)^{2}\right]\right] .
$$

For oxygen with normal coordination (total bond order $=2, \Delta_{i}^{e}=4$ ), Eq. (4) leads to two lone pairs. As the total bond order associated with a particular $\mathrm{O}$ atom starts to exceed 2, Eq. (4) causes a lone pair to gradually break up. This is accompanied by an energy penalty, as calculated by Eq. (5). $\Delta_{l p, i}$ in Eq. (5) depicts the deviation of the number of lone pairs around an atom from the number of lone pairs at normal coordination ( 2 for oxygen, 1 for nitrogen, 0 for carbon and hydrogen):

$$
E_{l p}=\frac{p_{l p} \Delta_{l p, i}}{1+\exp \left(-75 \Delta_{l p, i}\right)}
$$

To further account for the influence of lone pairs on molecular stability the energy expressions used in $\operatorname{ReaxFF}_{C H}$ to calculate the contributions of overcoordination energy $\left(\mathrm{E}_{\text {over }}\right)$ have been expanded to account for deviations in the number of lone pairs. The overcoordination for ReaxFF $F_{\text {nitro }}$ differs from that for $\mathrm{ReaxFF}_{C H}$ in that the overcoordination penalty gets diminished for systems like $\mathrm{H} 2 \mathrm{C}=\mathrm{N}=\mathrm{N}$ and
$\mathrm{R}-\mathrm{NO} 2$ which contain an over coordinated atom as a result of breaking up a formal lone electron pair $\left(\Delta_{l p, i}=1\right)$ next to a formally undercoordinated atom.

Valence angle conjugation. In simple valence bond theory, this is described in terms of a formal charge transfer leading to an $\mathrm{N}+$ atom that can make four bonds and for $\mathrm{H} 2 \mathrm{C}=\mathrm{N}=\mathrm{N}$ an $\mathrm{N}-$ atom that can make only two bonds. ReaxFF ${ }_{C H}$ contained a conjugation term keyed to torsion angles. This accounted for the effect of conjugation on rotational barriers. However, for nitro systems the main effect of $\mathrm{NO}_{2}$-conjugation is on the stability of the system and as such cannot describe the $\mathrm{NO}_{2}$-conjugation. To properly describe the stability of the nitro group we now include a conjugation term keyed to valence angles $\left(E_{\text {coa }}\right)$ to the ReaxFF energy description. Equation (6) shows the potential function used to describe the valence angle conjugation contribution in angle $i j k$ :

$$
\begin{aligned}
E_{c o a}= & p_{i j k}^{c o a} \frac{2+\exp \left(-\lambda_{21} \Delta_{j}\right)}{1+\exp \left(-\lambda_{21} \Delta_{j}\right)+\exp \left(\lambda_{22} \Delta_{j}\right)} \\
& \times \exp \left[-\lambda_{20}\left(\mathrm{BO}_{i j}-2\right)\right] \exp \left[-\lambda_{20}\left(\mathrm{BO}_{j k}-2\right)\right],
\end{aligned}
$$

where $\Delta_{j}$ describes the overcoordination on the central atom in valence angle $i j k$.

Terminal triple bond stabilization. While ReaxFF recognizes triple bonds, a bond order of 3.0 is attained at distances shorter than equilibrium since it reflects an asymptotic bond order value. At equilibrium, a system with a formal triple bond usually has a bond order of about 2.7. Although the atoms involved in the triple bond do not completely fill their valency, this loss of bonding is countered for systems with internal triple bonds by undercoodination stabilization allowing ReaxFF to properly describe $\mathrm{C} \equiv \mathrm{C}$ bonds. However, the approach used in $\operatorname{ReaxFF}_{C H}$ leads to too little stability in cases with terminal triple bonds as allowed by $\mathrm{N}$ and $\mathrm{O}$ systems $(\mathrm{HC} \equiv \mathrm{N}, \mathrm{C} \equiv \mathrm{O}$, and $\mathrm{N} \equiv \mathrm{N})$. Fortunately, it is straightforward to detect terminal triple bonds allowing us to use Eq. (7) to account for the additional stabilization:

$$
\begin{aligned}
E_{\text {trip }}= & \nu_{\text {trip }} \exp \left[-\lambda_{8}\left(\mathrm{BO}_{i j}-2.5\right)^{2}\right] \\
& \times \frac{1}{1+25 \exp \left[\lambda_{5}\left(\Delta_{i}+\Delta_{j}\right)\right]} \\
& \times\left[\exp \left[-\lambda_{4}\left(\mathrm{SBO}_{i}-\mathrm{BO}_{i j} j\right)\right]\right. \\
& \left.+\exp \left[-\lambda_{4}\left(\mathrm{SBO}_{j}-\mathrm{BO}_{i} j\right)\right]\right],
\end{aligned}
$$

$\mathrm{SBO}_{i}$ and $\mathrm{SBO}_{j}$ are the sum of the bond orders around atoms $i$ and $j$ and $\Delta_{i}$ and $\Delta_{j}$ describe the overcoordination in atoms $i$ and $j$, by subtracting the valency of these atoms from $\mathrm{SBO}_{i}$ and $\mathrm{SBO}_{j}$.

All the modifications introduced in $\mathrm{ReaxFF}_{\text {nitro }}$ are fully compatible with $\mathrm{ReaxFF}_{C H}$ and with the ReaxFF potentials for silicon/silicon oxides ${ }^{18}$ and aluminum/aluminum oxides. $^{19}$

\section{B. Parametrization of ReaxFF}

The parameters of the nitramine ReaxFF are based on a large number of ab initio QM calculations. Over 40 reactions 


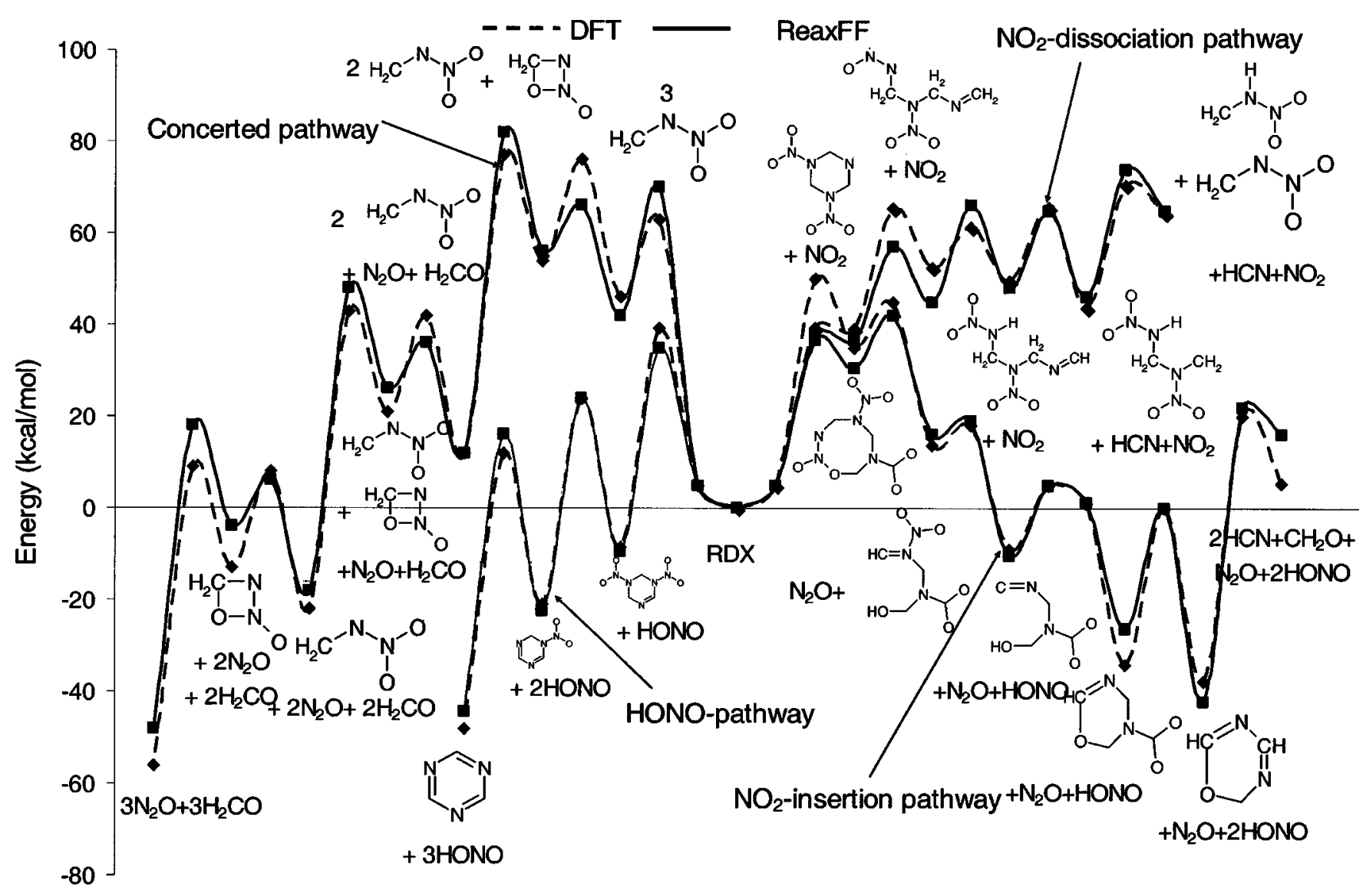

FIG. 1. Energetics of unimolecular decomposition mechanisms in RDX obtained using the ReaxFF (full lines with filled symbols) and with QM (dashed lines with open symbols) (Ref. 20). Circles represent the sequential HONO elimination, triangles show the decomposition process following homolytic N-N bond breaking $\left(\mathrm{NO}_{2}\right.$ elimination), and diamonds represent the concerted ring-opening pathway. Intermediates and products are described in Ref. 20.

and over 1600 equilibrated molecules have been used; they are designed to characterize the atomic interactions under various environments [likely and unlikely (high energy)] each atom can encounter. The training set contains bond breaking and compression curves for all possible bonds, angle and torsion bending data for all possible cases, as well as crystal data. The data used to parametrize ReaxFF ${ }_{\text {nitro }}$ includes all the cases previously used in the hydrocarbon training set described in Ref. 4 plus the additional cases detailed in the supplementary material of Ref. 5 which also compares the accuracy of ReaxFF in fitting the QM data.

Thus ReaxFF $F_{\text {nitro }}$ accurately describes both the gas phase chemistry of hydrocarbons and various $\mathrm{C}, \mathrm{H}, \mathrm{N}, \mathrm{O}$ systems, including the derived decomposition pathways of RDX. Figure 1 shows ReaxFF (full lines) and QM (dashed lines) results of the energetics of 21 intermediate and transition states involved in the four most important gas phase decomposition mechanisms. We show the main three mechanisms studied by Chakraborty ${ }^{20}$ [sequential HONO elimination, homolytic cleavage of an $\mathrm{NN}$ bond $\left(\mathrm{NO}_{2}\right.$ elimination) and subsequent decomposition, and concerted decomposition] as well as a pathway involving $\mathrm{NO}_{2}$ insertion suggested by Zhang et al. for HMX. ${ }^{21}$ For this last case we performed QM simulations at the same level used by Chakraborty et al. for the RDX-equivalent mechanism. We see from Fig. 1 that ReaxFF accurately describes the complex gas phase decomposition of RDX.
ReaxFF also describes crystal data accurately; we obtain lattice parameters of $a=13.7781 \AA, b=12.0300 \AA$, and $c$ $=10.9609 \AA$ in good agreement with the experimental values $(13.182,11.574$, and $10.709 \AA) .{ }^{22}$ The calculated bulk modulus $B_{T}=13.90 \mathrm{GPa}$ is also in good agreement with the experimental measurements that range from $13.0 \mathrm{GPa}^{23}$ (isothermal) to 11.1-11.4 $\mathrm{GPa}^{24}$ (isentropic, from ultrasonic resonant frequencies). Both lattice parameters and bulk modulus were obtained by fitting Rose's equation of state ${ }^{25}$ to energy-volume data obtained from isothermal, isocoric (NVT) MD at $T=300 \mathrm{~K}$. The lattice parameters for the NVT simulations were obtained by homogeneously scaling those resulting from energy minimization (relaxing atomic positions and lattice parameters).

\section{MOLECULAR DYNAMICS SIMULATIONS OF RDX THERMAL DECOMPOSITION}

We study the decomposition of RDX at various temperatures between $T=1200 \mathrm{~K}$ and $T=3000 \mathrm{~K}$ and at three densities: at $1.68 \mathrm{~g} / \mathrm{cm}^{3}$ (close to normal density-corresponding to zero pressure volume: $V=V_{0}$ ), under compression (2.11 $\left.\mathrm{g} / \mathrm{cm}^{3}\right)$ and at low density $\left(0.21 \mathrm{~g} / \mathrm{cm}^{3}\right)$ using the reactive potential ReaxFF with MD. The initial state of the simulations consist of RDX perfect crystals using simulation cells containing eight molecules (one unit cell, 168 atoms) and 3-D periodic conditions. After relaxing the atomic positions 
at each density with low temperature $\mathrm{MD}(T=10 \mathrm{~K})$ we study the time evolution of the system at the desired temperature with isothermal isochoric (NVT ensemble) MD simulations (using a Berendsen thermostat; the time scale associated with the coupling between the thermostat and the atomistic system was set to $200 \mathrm{fs}$ ). In order to correctly describe the fast chemistry under extreme conditions we use a time step of $0.1 \mathrm{fs}$ in integrating the equations of motion (nonreactive simulations can typically use one order of magnitude larger integration step).

Using just a single unit cell facilitated the exploration of lower temperatures processes where simulations for several nanoseconds were necessary to reach an equilibrium distribution of products (one nanosecond corresponds to ten million MD steps). We consider that use of one unit cell (eight molecules) is sufficient to characterize the overall time evolution of the chemistry during cook-off. In order to test system size dependence of our results we performed some simulations with larger cells $(2 \times 2 \times 2$ unit cells, $64 \mathrm{RDX}$ molecules, and 1344 atoms). Both time scales and equilibrium distribution of products are in excellent agreement with the smaller simulations.

Identifying chemical processes under the extreme conditions characteristic of $\mathrm{HE}$ decomposition is very challenging due to the high energies and fast time scales involved. In order to discuss these chemical processes in terms of molecules we must first define what we understand to be a molecule which in turn is based on the definition of bond. Bonds are usually defined in configuration space (positions): when two atoms are closer than some cutoff distance (depending on the elements involved) they belong to the same molecule. However, under the extreme conditions of temperature and pressure studied here two atoms may be close in configurational space for times shorter than a vibrational period (if their c.m. kinetic energy is larger than the binding energy). Thus, we use an alternative definition that two atoms are bonded if they are close in phase space (atomic positions and momenta); in practical terms we require the two atoms to have negative relative energy:

$$
E_{i j}=V_{i n t}^{(i j)}\left(r_{i j}\right)+K_{c m}^{(i j)}<0,
$$

where $V_{i n t}^{(i j)}\left(r_{i j}\right)$ is the effective pairwise interaction between atoms $i$ and $j$ separated by a distance $r_{i j}$ and $K_{c m}^{(i j)}$ is their c.m. kinetic energy. The interaction between two atoms in ReaxFF depends very strongly on their environment; for the purpose of defining bonds we estimate $V_{i n t}^{(i j)}(r)$ using an effective two body interaction with a modified Morse term:

$$
V_{i n t}^{(i j)}(r)=\left\{\begin{array}{l}
D_{i j}\left(\chi^{2}(r)-2 \chi(r)\right) \quad \text { if } r>R_{i j}, \\
-D_{i j} \quad \text { if } r<R_{i j},
\end{array}\right.
$$

where $\chi(r)=\exp \left[\gamma / 2\left(1-r / R_{i j}\right)\right]$. In order to define unbiased parameters for Eq. (9) the energy of the effective interactions $\left(D_{i j}\right)$ are proportional to the ReaxFF bond energy parameter ${ }^{4}$ and the distances $\left(R_{i j}\right)$ are proportional to the $\sigma$ bond distances. ${ }^{4}$ The three free parameters [energy scale, distance scale, and curvature $(\gamma)$ ] were chosen to correctly recognize RDX molecules in their crystalline form at $T$ $=300 \mathrm{~K}$. The prefactor used to define $D_{i j}$ from bond ener-
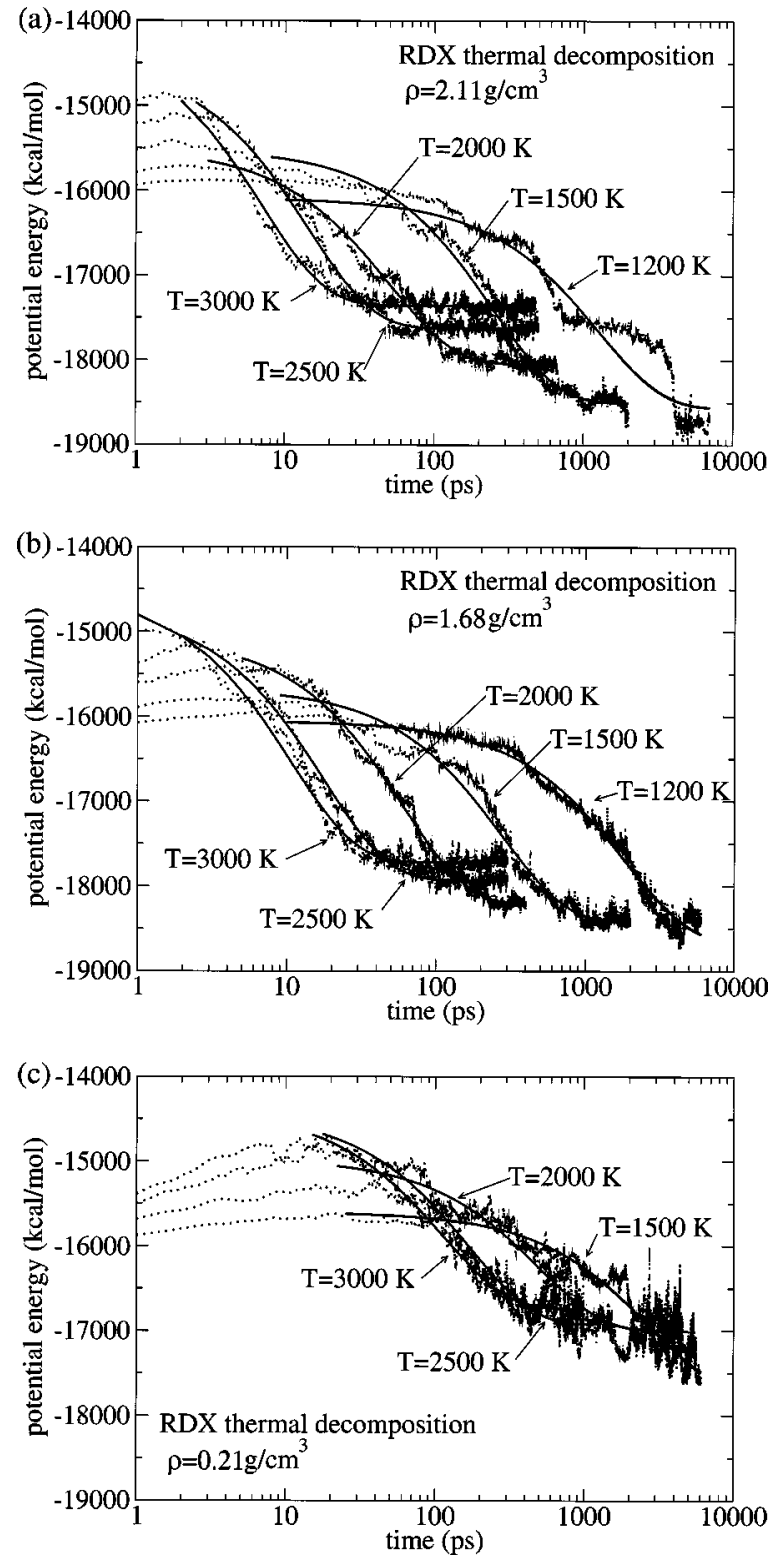

FIG. 2. Time evolution of potential energy (in $\mathrm{kcal} / \mathrm{mol}$ per unit cell) for temperatures from 1200 to $\mathrm{T}=3000 \mathrm{~K}$ and volumes $\mathrm{V}=0.8 \mathrm{~V}_{0}(\mathrm{a}), \mathrm{V}=\mathrm{V}_{0}$ (b), and $\mathrm{V}=8 \mathrm{~V}_{0}$ (c).

gies is $\frac{1}{3}$; this leads roughly to the energy of the $\sigma$ bonds. ${ }^{4}$ The distance parameters are obtained by multiplying the $\sigma$ bond distances by 1.35 ; this leads to equilibrium bond distances. In order to avoid having different molecules in the compressed crystals appear to be clustered together we chose a large value for the curvature $\gamma=25$. Similar cluster recognition methods have been used in fragmentation. ${ }^{26}$

\section{ENERGETICS AND TIME SCALES OF DECOMPOSITION}

Figure 2 shows the time evolution of the potential energy of RDX for temperatures $T=1200,1500,2000,2500$, and $3000 \mathrm{~K}$ at three densities: under compression $\left(2.11 \mathrm{~g} / \mathrm{cm}^{3}\right)$ [Fig. 2(a)], for normal density $\left(1.68 \mathrm{~g} / \mathrm{cm}^{3}\right)$ [Fig. 2(b)], and for the low density case $\left(0.21 \mathrm{~g} / \mathrm{cm}^{3}\right)$ [Fig. 2(c)]. It is clear see from Fig. 2 that higher densities lead to faster chemistry. 
TABLE I. Parameters describing the exponential behavior of potential energy with time during RDX decomposition obtained from our MD simulations as a function of temperature $(T)$ and density $(\rho)$. We also show the equilibration-induction time for each case $\left(t_{T-I}\right)$. Energies are given in $\mathrm{kcal} / \mathrm{mol}$ and times in $\mathrm{ps}$.

\begin{tabular}{lccccr}
\hline \hline$T(\mathrm{~K})$ & $\rho\left(\mathrm{g} / \mathrm{cm}^{3}\right)$ & $t_{T-I}$ & $U_{0}$ & $\Delta U$ & \multicolumn{1}{c}{$\tau$} \\
\hline 1200 & 2.11 & 10 & -18565.9 & 2475.66 & 1260.18 \\
1500 & 2.11 & 8 & -18502.1 & 2992.04 & 248.28 \\
2000 & 2.11 & 3 & -18043.6 & 2541.12 & 50.44 \\
2500 & 2.11 & 2.5 & -17622.8 & 3160.93 & 14.47 \\
3000 & 2.11 & 2 & -17355.0 & 3217.71 & 6.94 \\
1200 & 1.68 & 10 & -18667.6 & 2610.23 & 1857.16 \\
1500 & 1.68 & 9 & -18423.3 & 2759.97 & 276.96 \\
2000 & 1.68 & 5 & -18213.3 & 3150.71 & 59.96 \\
2500 & 1.68 & 2 & -17931.4 & 3218.99 & 17.73 \\
3000 & 1.68 & 1 & -17720.0 & 3201.26 & 10.73 \\
1500 & 0.21 & 25 & -17705.5 & 2102.23 & 2688.19 \\
2000 & 0.21 & 22 & -17004.9 & 2032.99 & 498.93 \\
2500 & 0.21 & 17.5 & -16929.8 & 2494.95 & 169.34 \\
3000 & 0.21 & 15.0 & -16743.3 & 2347.87 & 112.44 \\
\hline \hline
\end{tabular}

After an initial equilibration and induction time (denoted $\left.t_{E-I}\right)$ the potential energy decreases with time as the chemical reactions progress. Its temporal evolution can then be described rather well with a simple exponential function:

$$
\begin{aligned}
U(t ; T, \rho)= & U_{0}(T, \rho)+\Delta U(T, \rho) \\
& \times \exp \left[-\left(t-t_{E-I}\right) / \tau(T, \rho)\right],
\end{aligned}
$$

where $U_{0}$ is the asymptotic energy of the products, $\Delta U$ is the exothermicity of the reaction, and $\tau$ is an overall characteristic time of reaction; all three parameters are temperature and density dependent. In Table I we show the parameters obtained from fitting Eq. (10) to our MD data.

Figure 3 shows the characteristic times (in logarithmic scale) as a function of inverse temperature (Arrhenius plot). The dashed line corresponds to MD results at $2.11 \mathrm{~g} / \mathrm{cm}^{3}$, the solid line with open squares corresponds to $1.68 \mathrm{~g} / \mathrm{cm}^{3}$, and the dotted one represents the theoretical results $0.21 \mathrm{~g} / \mathrm{cm}^{3}$. The solid line shows the behavior of HMX (an HE material

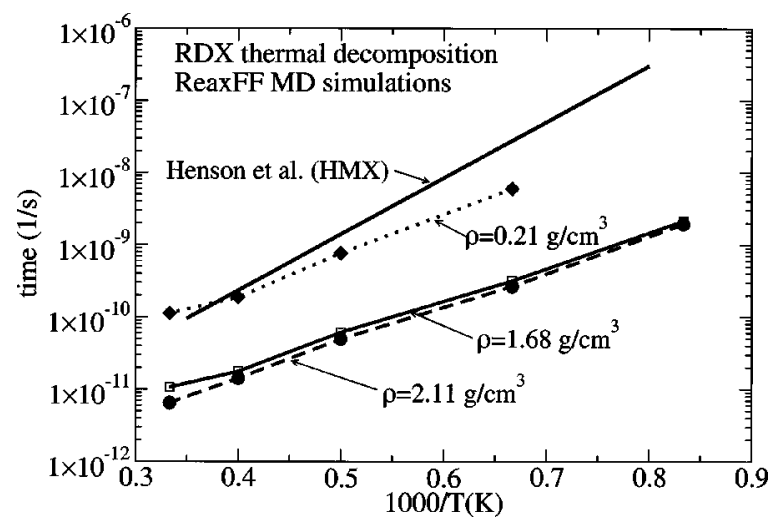

FIG. 3. Characteristic time versus inverse temperature from ReaxFF MD simulations for different volumes: $\mathrm{V}=0.8 \mathrm{~V}_{0}$ (red line), $\mathrm{V}=\mathrm{V}_{0}$ (black line), and $\mathrm{V}=8 \mathrm{~V}_{0}$ (green line). We also include the Arrhenius behavior obtained from a wide range of experimental ignition times in HMX (blue line) (Ref. 27). very similar to RDX) obtained from a wide variety of experimental ignition times, including fast processes $\left(10^{-9} \mathrm{~s}\right)$, by Henson and collaborators. ${ }^{27}$ The compilation of experimental data includes a wide variety of sources such as thermal explosions, fast pyrolysis, laser heating, and detonations. ${ }^{27}$ Figure 3 shows that our first principles-based calculations are in reasonable quantitative agreement with experiments. The Arrhenius behavior shown by both MD and experimental results can be attributed to a single rate-limiting step in the sequence of reactions that lead to decomposition. From the MD simulations we obtain an activation energy $\left(E_{a}\right)$ for this limiting step that increases as the density decreases: for $\rho=2.11 \mathrm{~g} / \mathrm{cm}^{3}$ we obtain $E_{a} \sim 23 \mathrm{kcal} / \mathrm{mol}$ and for $\rho=0.21$ $\mathrm{g} / \mathrm{cm}^{3} E_{a}=26.6 \mathrm{kcal} / \mathrm{mol}$.

\section{TIME EVOLUTION OF INTERMEDIATES AND PRODUCTS}

In Fig. 4 we show the time evolution of the population of key products $\mathrm{N}_{2}, \mathrm{H}_{2} \mathrm{O}, \mathrm{CO}$, and $\mathrm{CO}_{2}$ and intermediate $\mathrm{NO}_{2}$; the time is scaled with the corresponding characteristic reaction time obtained from the energy evolution. We find that $\mathrm{N}-\mathrm{N}$ bond breaking to form $\mathrm{NO}_{2}$ is the dominant early chemical reaction for the conditions studied. This occurs despite the similar energy barrier for HONO elimination. This difference is expected since the $\mathrm{NO}_{2}$ formation mechanism leads to greatly increased entropy (and hence less free energy), while HONO elimination has a tight transition state (low entropy and high free energy). This was also observed in the pressure and temperature dependence in the reaction rates estimated in Ref. 28. It was also apparent in the shock induced decomposition ${ }^{5}$ whereas $\mathrm{NO}_{2}$ products dominate the early species for weak shocks (up to particle velocity of 3 $\mathrm{km} / \mathrm{s}$ ) while higher velocities led to equal amounts of $\mathrm{HO}$ and $\mathrm{NO}$ at early times. For every condition of temperature and density we find that the time scales of $\mathrm{H}_{2} \mathrm{O}$ and $\mathrm{N}_{2}$ formation are similar to one another and to the overall characteristic time $(\tau)$; formation of $\mathrm{H}_{2} \mathrm{O}$ and $\mathrm{N}_{2}$ occurs during the early stages of the process. Manaa et al. found that $\mathrm{H}_{2} \mathrm{O}$ forms faster than $\mathrm{N}_{2}$ in HMX decomposition using ab initio MD. We also find that the time scales of $\mathrm{CO}$ and $\mathrm{CO}_{2}$ formation depend on density: for normal and high density the time scales of $\mathrm{CO}$ and $\mathrm{CO}_{2}$ formation are larger than those of $\mathrm{H}_{2} \mathrm{O}$ and $\mathrm{N}_{2}$ whereas at low density $\mathrm{CO}$ forms promptly and with time scales similar to those of $\mathrm{H}_{2} \mathrm{O}$ and $\mathrm{N}_{2}$ (see Fig. 4).

\section{EQUILIBRIUM STATE OF THE PRODUCTS}

\section{A. Asymptotic population of key molecules}

Figure 5 shows the asymptotic (time averaged) populations of the key products $\left(\mathrm{N}_{2}, \mathrm{H}_{2} \mathrm{O}, \mathrm{CO}\right.$, and $\left.\mathrm{CO}_{2}\right)$ for the various temperatures and densities studied. We find that the population of $\mathrm{N}_{2}$ decreases with compression. At low density essentially all $\mathrm{N}$ atoms form $\mathrm{N}_{2}$ molecules; for $\rho=2.11 \mathrm{~g} / \mathrm{cm}^{3}$ the population of $\mathrm{N}_{2}$ is smaller (involving roughly half the $\mathrm{N}$ atoms) and increases with temperature. The population of $\mathrm{H}_{2} \mathrm{O}$ also decreases under compression but the values for $\rho=0.21$ and $1.68 \mathrm{~g} / \mathrm{cm}^{3}$ are comparable. The populations of $\mathrm{CO}$ and $\mathrm{CO}_{2}$ show a much more marked density dependence. We see from Fig. 5 that at low densities $\left(\rho=0.21 \mathrm{~g} / \mathrm{cm}^{3}\right)$ 

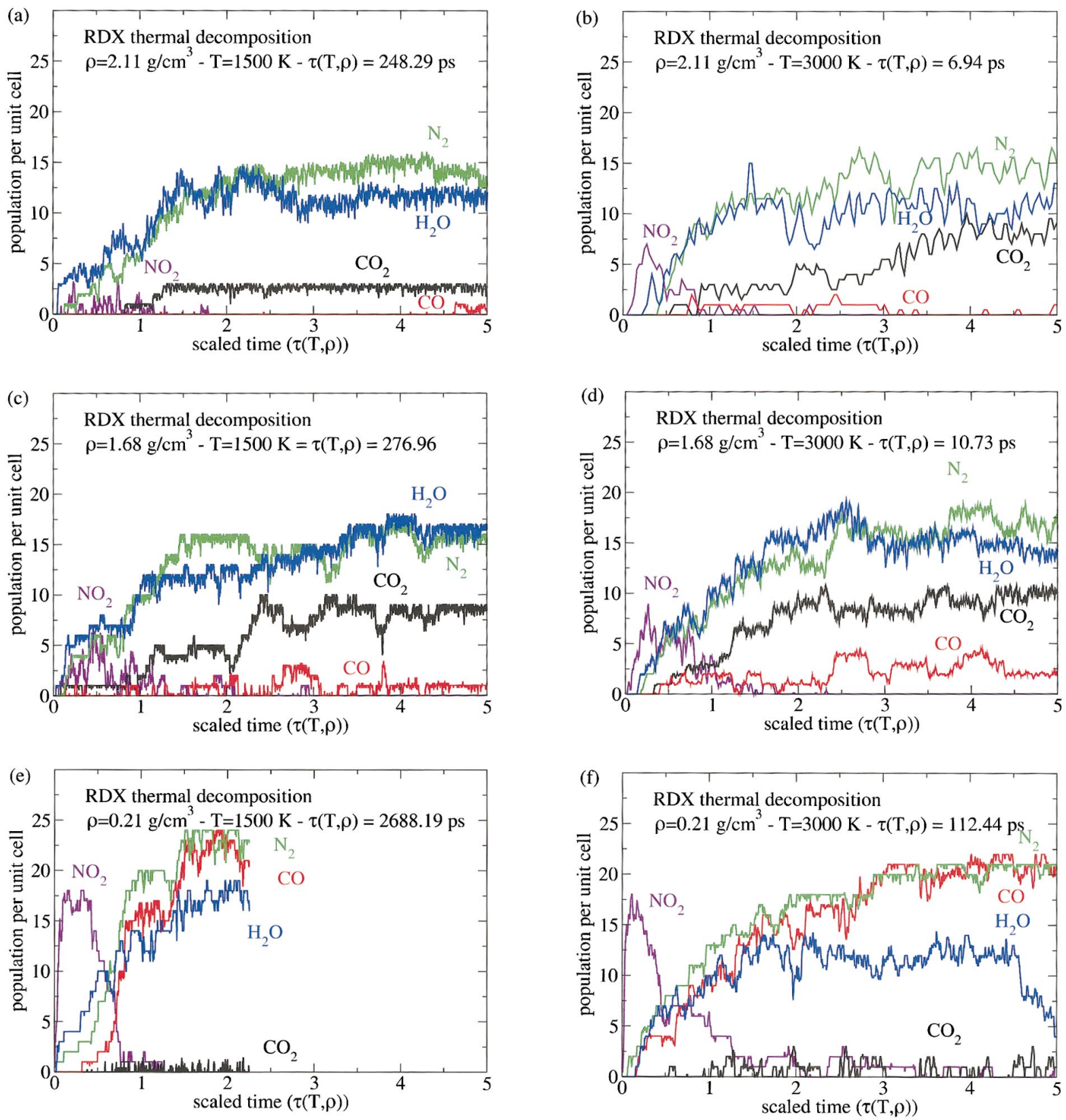

FIG. 4. (Color) Time evolution of products $\mathrm{N}_{2}, \mathrm{H}_{2} \mathrm{O}, \mathrm{CO}$, and $\mathrm{CO}_{2}$ and intermediate $\mathrm{NO}_{2}$ for $\mathrm{T}=3000 \mathrm{~K}$ and $\mathrm{T}=1500 \mathrm{~K}$ and three densities $\rho=2.11 \mathrm{~g} / \mathrm{cm}^{3}$, $\rho=1.68 \mathrm{~g} / \mathrm{cm}^{3}$, and $\rho=0.21 \mathrm{~g} / \mathrm{cm}^{3}$. Here (b) corresponds approximately to the CJ point.

virtually all $\mathrm{C}$ atoms form $\mathrm{CO}$ molecules while we find no $\mathrm{CO}_{2}$ molecules (the total number of $\mathrm{C}$ atoms in the system is 24). Figure 6(a) shows a snapshot of the equilibrium state for $T=3000 \mathrm{~K}$ and $\rho=0.21 \mathrm{~g} / \mathrm{cm}^{3}$. All atoms form small molecules; in addition to the ones shown in Fig. 5 we find $\mathrm{O}_{2}$ and $\mathrm{H}_{2}$ with asymptotic populations of 4 and 7 , respectively. As the density increases the equilibrium population of $\mathrm{CO}_{2}$ increases at the expense of $\mathrm{CO}$ molecules; this happens because at higher densities $\mathrm{C}$ atoms tend to clusterize into condensed phase aggregates, leading to a $\mathrm{C}$-poor gas phase. This results in an increase in the population of $\mathrm{CO}_{2}$ molecules. For $\rho=2.11 \mathrm{~g} / \mathrm{cm}^{3}$ we find almost no CO molecules; all the C atoms that do not form $\mathrm{CO}_{2}$ molecules end up in the condensed phase aggregate. Figure 6(b) shows a snapshot of an equilibrium configuration for $V=0.8 V_{0}$, containing a small condensed phase cluster with ten carbon atoms.

The phenomena of carbon clustering is very important to understanding the properties of HE materials. In Fig. 7 we plot the maximum number of $\mathrm{C}$ atoms in a single molecule as a function of temperature and for the three densities studied here. For $\rho=0.21 \mathrm{~g} / \mathrm{cm}^{3}$ there are no molecules with more that one $\mathrm{C}$ atom at any temperature. For $\rho=1.68 \mathrm{~g} / \mathrm{cm}^{3}$ and high temperatures the largest carbon aggregate slowly grows with decreasing temperature, but between $T=1500$ and $1200 \mathrm{~K}$ we find an abrupt jump in the size of the condensed phase. For $\rho=2.11 \mathrm{~g} / \mathrm{cm}^{3}$ we find larger C clusters at all temperatures and the abrupt increase in the size of the $\mathrm{C}$ aggregate occurs at higher temperatures (between 2000 and 

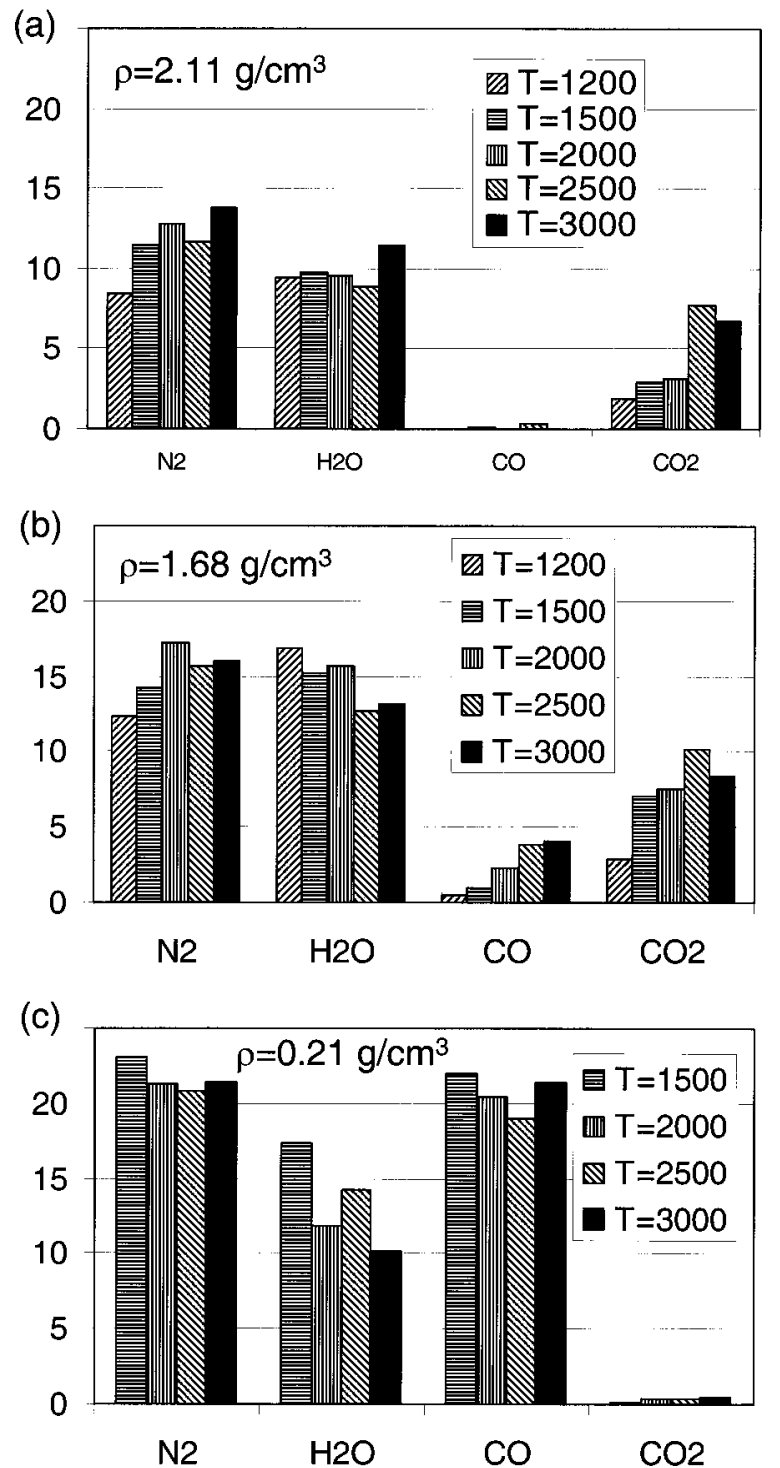

FIG. 5. Final population of key molecules for various temperatures from $\mathrm{T}=3000 \mathrm{~K}$ to $\mathrm{T}=1200 \mathrm{~K}$ and three densities: $\rho=2.11 \mathrm{~g} / \mathrm{cm}^{3}$ (a), $\rho=1.68$ $\mathrm{g} / \mathrm{cm}^{3}$ (b), and $\rho=0.21 \mathrm{~g} / \mathrm{cm}^{3}$ (c).

$2500 \mathrm{~K})$. Larger systems and longer simulations are necessary to fully characterize the relatively slow process of carbon clustering, nevertheless our simulations seem to indicate the existence of two distinct regimes: one dominated by large carbon aggregates (favored by high density and low temperature) and a second in which most $\mathrm{C}$ atoms form small molecules (for low densities and high temperatures).

\section{B. Mean lifetimes of products in equilibrium}

Figures 4 and 5 show total populations of various molecules but do not provide any information regarding their atomic stability: what is the average lifetime of an individual molecule? Under these extreme conditions we expect populations to be in dynamical equilibrium by undergoing frequent atomic rearrangements.

A direct calculation of the mean lifetime of a given molecule type would involve averaging the time elapsed between the formation of a given molecule and its disappear-
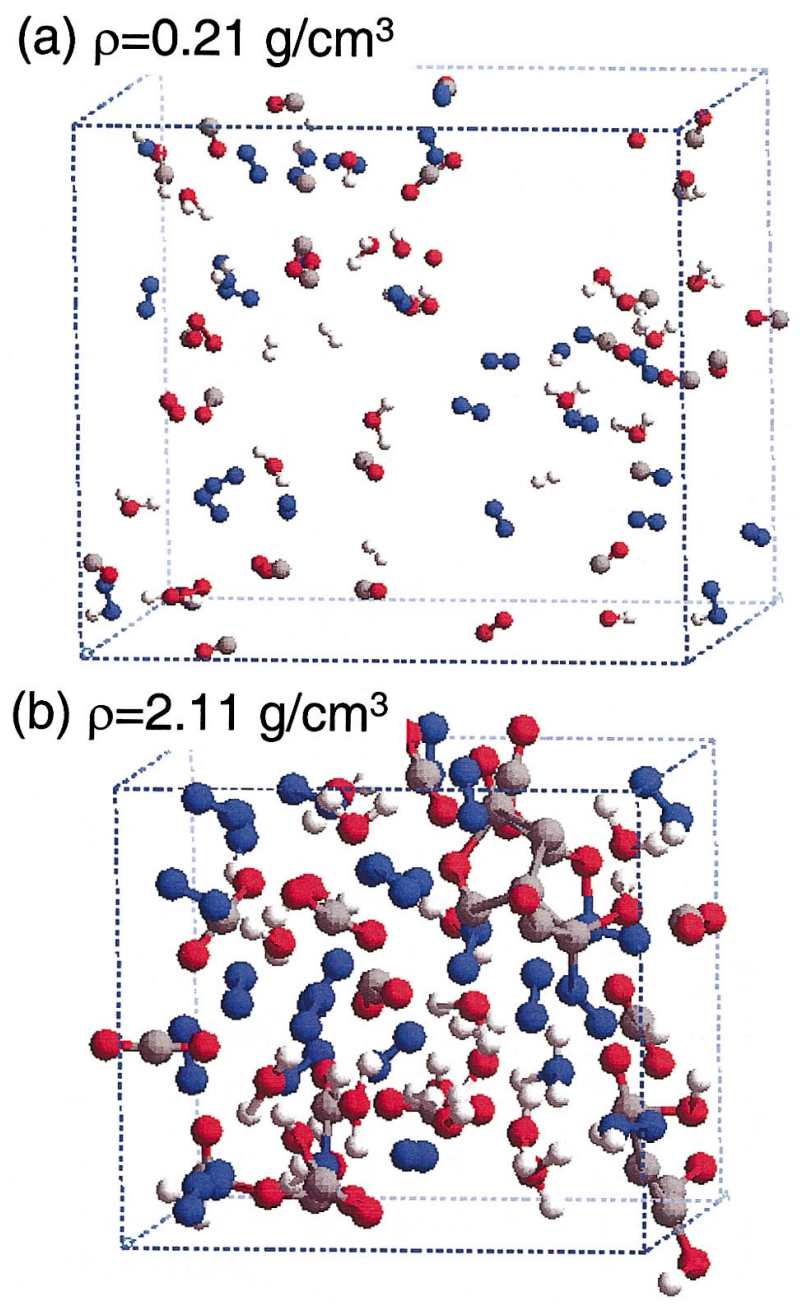

FIG. 6. (Color) (a) Snapshot of an equilibrium configuration for $\mathrm{T}=3000 \mathrm{~K}$ and $\rho=0.21 \mathrm{~g} / \mathrm{cm}^{3}$. (b) Snapshot for $\mathrm{T}=3000 \mathrm{~K}$ and $\rho=2.11 \mathrm{~g} / \mathrm{cm}^{3}$; this corresponds approximately to the CJ conditions, showing molecular character at this point.

ance. This direct method has two drawbacks: First, it leads to very inaccurate results when the mean lifetime is longer than or comparable to the simulation time. The second problem is that it is difficult to recognize unambiguously molecules in a

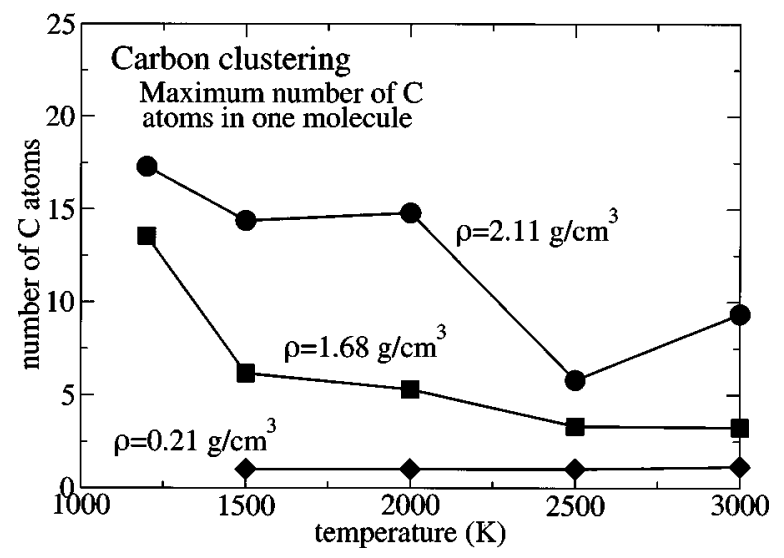

FIG. 7. Maximum number of $\mathrm{C}$ atoms in a single molecule as a function of temperature for $\rho=2.11 \mathrm{~g} / \mathrm{cm}^{3}$ (squares), $1.68 \mathrm{~g} / \mathrm{cm}^{3}$ (circles), and 0.21 $\mathrm{g} / \mathrm{cm}^{3}$ (diamonds). 
single snapshot in time of atomic positions and velocities. A molecule may seem to disappear (large vibrational amplitude) and reappear shortly after (within one or two vibrational periods). Is one to count such an event as a real chemical reaction or should it be considered an artifact and discarded? The molecule recognition algorithms used in MD have limitations in properly accounting for such effects, leading to serious problems in calculating mean lifetimes.

We use a more robust method to estimate the mean lifetime of molecules from MD simulations based on the fact that in most cases several statistically equivalent molecules of the same type are present at any given time. We find it convenient to evaluate the mean lifetimes $\left(T_{\alpha}\right)$ of molecule $\alpha$ (once its population reached its asymptotic values) using

$$
T_{\alpha}=\frac{\Delta t N_{\alpha}}{2\left(N_{\alpha}^{\prime}-N_{\alpha}\right)}
$$

where $\Delta \mathrm{t}$ is the period of time used in the calculation, $N_{\alpha}$ is the average number of molecules of type $\alpha$, and $N_{\alpha}^{\prime}$ is the number of different (atomistically distinguishable) molecules of type $\alpha$ that were present during the time of observation. The numerator in Eq. (11) represents to total effective observation time; the denominator represents the number of times a molecule of type $\alpha$ is created or destroyed. If $N_{\alpha}^{\prime}=N_{\alpha}$, no reactions occurred during the observation time and our best estimate of the mean lifetime is infinity. As another example of the meaning of Eq. (11), suppose we start the calculation with ten molecules of a given type and one of them disappears while a new one is formed during the observation time $(\Delta t)$; the average number of molecules $(N)$ is ten and the number of distinguishable molecules is 11 . The mean lifetime according to Eq. (11) is $T=10 \Delta t / 2$. This is the result we would obtain if we observed a single molecule for a time $10 \Delta t$ and our molecule disappears and a new one is formed during the observation time. Note that Eq. (11) is only valid once the molecular population has reached its asymptotic value; if more molecules of type $\alpha$ are being formed than destroyed, Eq. (11) will underestimate their mean lifetime.

Figure 8 shows the mean free lifetimes for $\mathrm{N}_{2}, \mathrm{H}_{2} \mathrm{O}$, $\mathrm{CO}$, and $\mathrm{CO}_{2}$ as a function of inverse temperature for the three densities studied. Note that the populations of $\mathrm{CO}$ at $\rho=2.11 \mathrm{~g} / \mathrm{cm}^{3}$ and that of $\mathrm{CO}_{2}$ at $\rho=0.21 \mathrm{~g} / \mathrm{cm}^{3}$ are very small and the calculation of mean lifetime becomes very inaccurate. Even for the other cases the statistical uncertainties (due to small system size and short time-scales characteristic of MD simulation) are significant. Our MD simulations show that for all conditions studied we have well defined molecules with mean lifetimes between $\sim 10 \mathrm{ps}$ and $10 \mathrm{~ns}$. The simulation at $\rho=2.11 \mathrm{~g} / \mathrm{cm}^{3}$ and $T=3000$ correspond to conditions close to those of the C-J point in RDX. We find that under those conditions the mean lifetime of $\mathrm{H}_{2} \mathrm{O}$ molecules is $\sim 5 \mathrm{ps}$, that of $\mathrm{CO}_{2}$ is around $30 \mathrm{ps}$, and $\mathrm{N}_{2}$ lives much longer, $\sim 450 \mathrm{ps}$. This indicates that at the C-J point the system is in a molecular state (rather than a plasma like state with no covalent bonding).
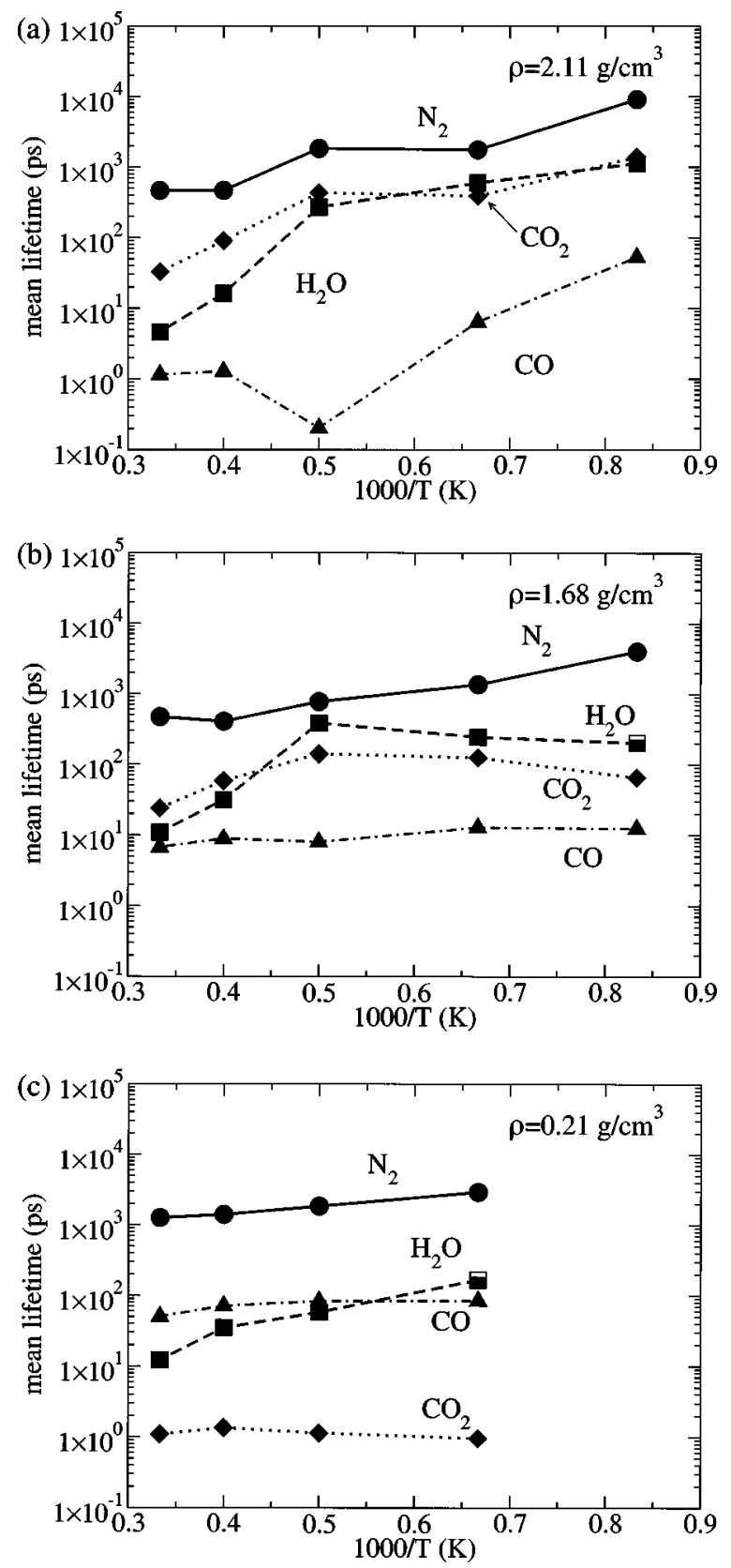

FIG. 8. Equilibrium mean life times of products [Eq. (11)] as a function of temperature for the three densities studied.

\section{CONCLUSIONS}

In summary, we studied the thermal decomposition of RDX for a variety of temperatures and densities using the first principles-based reactive force field ReaxFF with MD. From our simulations we extract a characteristic reaction time that shows Arrhenius temperature dependence and decreases with density. This suggests that a single step kinetics is a good approximation in this case. We also studied the time evolution and asymptotic population of various products and intermediates. The population of $\mathrm{CO}$ and $\mathrm{CO}_{2}$ show a marked density dependence. For low density essentially all $\mathrm{C}$ atoms form $\mathrm{CO}$ molecules, with an asymptotic population of $\mathrm{CO}_{2}$ of almost zero. With compression $\mathrm{C}$ atoms tend to clusterize into a condensed phase aggregate leading to a $\mathrm{C}$-poor gas phase and an increase in the population of $\mathrm{CO}_{2}$ at 
the expense of CO. For $\rho=2.11 \mathrm{~g} / \mathrm{cm}^{3}$ we find almost no CO molecules. Our simulations shed some light into the initial stages of the process of carbon clustering.

The molecular state of the material at CJ conditions is a critical question in the energetic materials community and very hard to answer experimentally. Our results show that under conditions near to the CJ point $\left(\rho=2.11 \mathrm{~g} / \mathrm{cm}^{3}\right.$ and $T$ $=3000 \mathrm{~K}$ ) molecules with relatively long mean lifetimes (in terms bond vibrations) are clearly identifiable: the mean lifetime of $\mathrm{N}_{2}$ molecules is around $300 \mathrm{ps}$, while $\mathrm{H}_{2} \mathrm{O}$ and $\mathrm{CO}_{2}$ have lifetimes of 5 and $30 \mathrm{ps}$, respectively. This shows that under CJ conditions RDX corresponds to a molecular state as opposed to being plasmalike with no molecular entities.

The accurate description of the shock velocity as a function of piston velocity in RDX shocks ${ }^{5}$ and the reasonable time scales for the thermal decomposition of RDX (as compared with experiments in a similar energetic material, HMX) indicate that ReaxFF yields an accurate description of the atomic interactions of RDX even under the extreme conditions attained during detonation.

Atomistic modeling with these new generation reactive potentials is becoming an increasingly important tool to investigate condensed-phase chemistry under dynamical or static loading. Such simulations provide very detailed information regarding the decomposition and subsequent reactions in energetic materials that allow one to make some sense out of the very complex mechanical and chemical processes. Furthermore, since ReaxFF is based on ab initio calculations, all the predictions in this paper can be considered as first principles (no critical empirical data was used in our calculations). Finally, the data presented in this paper should provide valuable input to mesoscopic and macroscopic models of energetic materials and these type of simulations are an important step to develop physics-based, predictive materials models.

\section{ACKNOWLEDGMENTS}

Work at Los Alamos National Laboratory was supported by the ASC Materials and Physics Modeling Program, LANL. Work at Caltech was supported by ONR (program manager Judah Goldwasser).
${ }^{1}$ D. D. Dlott, Annu. Rev. Phys. Chem. 50, 251 (1999).

${ }^{2}$ S. D. McGrane, D. S. Moore, and D. J. Funk, J. Appl. Phys. 93, 5063 (2003).

${ }^{3}$ D. W. Brenner, O. A. Shenderova, J. A. Harrison, S. J. Stuart, B. Ni, and S. B. Sinnott, J. Phys.: Condens. Matter 14, 783 (2002); S. J. Stuart, A. B. Tutein, and J. A. Harrison, J. Chem. Phys. 112, 6472 (2000)

${ }^{4}$ A. C. T. van Duin, S. Dasgupta, F. Lorant, and W. A. Goddard III, J. Phys. Chem. A 105, 9396 (2001)

${ }^{5}$ A. Strachan, A. C. T. van Duin, D. Chakraborty, S. Dasgupta, and W. A. Goddard III, Phys. Rev. Lett. 91, 098301 (2003).

${ }^{6}$ W. Ficket and W. C. Davis, Detonation (Univ. of California, Berkeley, 1979).

${ }^{7}$ M. L. Elert, D. M. Deaven, D. W. Brenner, and C. T. White, Phys. Rev. B 39, 1453 (1989)

${ }^{8}$ D. W. Brenner, D. H. Robertson, M. L. Elert, and C. T. White, Phys. Rev. Lett. 70, 2174 (1993).

${ }^{9}$ J. W. Mintmire, J. J. C. Barrett, D. H. Robertson, and C. T. White, Chem. Phys. Rep. 17, 37 (1998)

${ }^{10}$ B. L. Holian, T. C. Germann, J.-B. Maillet, and C. T. White, Phys. Rev. Lett. 89, 285501 (2002).

${ }^{11}$ C. T. White, D. H. Robertson, D. R. Swanson, and M. L. Elert, AIP Conf. Proc. 505, 377 (2000)

${ }^{12}$ B. M. Rice, W. Mattson, and S. F. Trevino, Phys. Rev. E 57, 5106 (1998).

${ }^{13}$ M. R. Manaa, L. E. Fried, C. F. Melius, M. Elstner, and Th. Frauenheim, J. Phys. Chem. A 106, 9024 (2002).

${ }^{14}$ J.-B. Maillet, M. Mareschal, L. Soulard, R. Ravelo, P. S. Lomdahl, T. C. Germann, and B. L. Holian, Phys. Rev. E 63, 016121 (2001).

${ }^{15}$ E. J. Reed, L. E. Fried, and J. D. Joannopoulos, Phys. Rev. Lett. 90, 235503 (2003)

${ }^{16}$ J. K. Brennan and B. M. Rice, Mol. Phys. 101, 3309 (2003).

${ }^{17}$ R. Ravelo, B. L. Holian, T. C. Germann, and P. S. Lomdahl, Phys. Rev. B 70, 014103 (2004).

${ }^{18}$ A. C. T. van Duin, A. Strachan, S. Stewman, Q. S. Zhang, X. Xu, and W. A. Goddard III, J. Phys. Chem. 107, 3803 (2003).

${ }^{19}$ Q. Zhang, T. Cagin, A. C. T. van Duin, W. A. Goddard III, Y. Qi, and L. G. Hector, Phys. Rev. B 69, 045423 (2004).

${ }^{20}$ D. Chakraborty, R. P. Muller, S. Dasgupta, and W. A. Goddard III, J. Phys. Chem. A 104, 2261 (2000).

${ }^{21}$ S. Zhang, H. N. Nguyen, and T. N. Truong, J. Phys. Chem. A 107, 2981 (2003).

${ }^{22}$ C. S. Choi and E. Prince, Acta Crystallogr., Sect. B: Struct. Crystallogr. Cryst. Chem. B28, 2857 (1972).

${ }^{23}$ B. Olinger, B. Roof, and H. Cady, Symposium International Sur Le Comportment Des Milieux Denses Sous Hautes Pressions Dynamiques, Commissariat a l'Energie Atomique Centre d'Etudes de Vajours, Paris, France, 1978, p. 3.

${ }^{24}$ S. Haussül, Z. Kristallogr. 216, 339 (2001).

${ }^{25}$ J. H. Rose, J. Ferrante, and J. R. Smith, Phys. Rev. Lett. 47, 675 (1981).

${ }^{26}$ A. Strachan and C. O. Dorso, Phys. Rev. C 56, 995 (1997); S. Pratt, C. Montoya, and F. Romming, Phys. Lett. B 349, 261 (1995); J. Pan and S. D. Gupta, Phys. Rev. C 51, 1384 (1995).

${ }^{27}$ B. F. Henson, L. Smilowitz, B. W. Asay, P. M. Dickson, and P. M. Howe, Proceedings of the 12th Symposium (International) on Detonation (2002), http://www.sainc.com/detsymp/technicalProgram.htm

${ }^{28}$ D. Chakraborty, R. P. Muller, S. Dasgupta, and W. A. Goddard III, J. Comput.-Aided Mater. Des. 8, 203 (2001). 